\title{
OXYGEN ISOTOPIC RATIOS IN COOL R CORONAE BOREALIS STARS
}

\author{
D. A. García-Hernández ${ }^{1,2}$, David L. Lambert ${ }^{3}$, N. Kameswara RaO ${ }^{4}, \mathrm{Ken}$ H. Hinkle ${ }^{5}$, and KJell Eriksson ${ }^{6}$ \\ ${ }^{1}$ Instituto de Astrofísica de Canarias (IAC), C/Via Láctea s/n, 38200 La Laguna, Tenerife, Spain; agarcia@iac.es \\ ${ }_{2}^{2}$ Departamento de Astrofísica, Universidad de La Laguna (ULL), E-38205 La Laguna, Tenerife, Spain \\ ${ }^{3}$ W. J. McDonald Observatory. The University of Texas at Austin. 1 University Station, C1400. Austin, TX 78712-0259, USA; dll@astro.as.utexas.edu \\ ${ }^{4}$ Indian Institute of Astrophysics, Bangalore 560 034, India; nkrao@iiap.res.in \\ ${ }^{5}$ National Optical Astronomy Observatory (NOAO), Tucson, AZ 85726, USA; hinkle@noao.edu \\ ${ }^{6}$ Department of Physics and Astronomy, Uppsala University, Box 515, 75120 Uppsala, Sweden; Kjell.Eriksson@ astro.uu.se \\ Received 2010 February 25; accepted 2010 March 16; published 2010 April 7
}

\begin{abstract}
We investigate the relationship between R Coronae Borealis (RCB) stars and hydrogen-deficient carbon (HdC) stars by measuring precise ${ }^{16} \mathrm{O} /{ }^{18} \mathrm{O}$ ratios for five cool RCB stars. The ${ }^{16} \mathrm{O} /{ }^{18} \mathrm{O}$ ratios are derived by spectrum synthesis from high-resolution $(R \sim 50,000) K$-band spectra. Lower limits to the ${ }^{16} \mathrm{O} /{ }^{17} \mathrm{O}$ and ${ }^{14} \mathrm{~N} /{ }^{15} \mathrm{~N}$ ratios as well as $\mathrm{Na}$ and $\mathrm{S}$ abundances (when possible) are also given. RCB stars in our sample generally display less ${ }^{18} \mathrm{O}$ than $\mathrm{HdC}$ stars - the derived ${ }^{16} \mathrm{O} /{ }^{18} \mathrm{O}$ ratios range from 3 to 20 . The only exception is the RCB star WX CrA, which seems to be an HdC-like star with ${ }^{16} \mathrm{O} /{ }^{18} \mathrm{O}=0.3$. Our result of a higher ${ }^{16} \mathrm{O} /{ }^{18} \mathrm{O}$ ratio for the RCB stars must be accounted for by a theory of the formation and evolution of $\mathrm{HdC}$ and $\mathrm{RCB}$ stars. We speculate that a late dredge-up of products of He burning, principally ${ }^{12} \mathrm{C}$ and ${ }^{16} \mathrm{O}$, may convert an ${ }^{18} \mathrm{O}$-rich $\mathrm{HdC}$ star into an ${ }^{18} \mathrm{O}$-poor RCB star as the H-deficient star begins its final evolution from a cool supergiant to the top of the white dwarf cooling track.
\end{abstract}

Key words: infrared: stars - stars: abundances - stars: atmospheres - stars: chemically peculiar - white dwarfs

Online-only material: color figures

\section{INTRODUCTION}

The R Coronae Borealis ( $\mathrm{RCB}$ ) stars and their likely cousins, the hydrogen-deficient carbon (HdC) stars and the extreme helium (EHe) stars, have long posed a puzzle as to their origins in terms of stellar evolution. Two leading scenarios have survived decades of theoretical and observational scrutiny. In one, the $\mathrm{H}$-deficient supergiant is formed from the merger of a He white dwarf (WD) with a C-O WD (Webbink 1984; Iben \& Tutukov 1984; Saio \& Jeffery 2002). This path is widely referred to as the double-degenerate (DD) scenario. In the other, these H-deficient stars result from a final, post-asymptotic giant branch helium shell flash in the central star of a planetary nebula. The final flash may transform the star on the WD cooling track into an $\mathrm{H}$-deficient supergiant; this the so-called born-again scenario is discussed by Herwig (2001) and Blöcker (2001) and often labeled the final flash (FF) scenario. Remaining open questions include: is the DD or the FF scenario the dominant mechanism? If both are operative, how does one distinguish a product of the DD from the one of the FF scenario?

Elemental and isotopic abundances of $\mathrm{C}, \mathrm{N}$, and $\mathrm{O}$ are potentially powerful agents for testing the different evolutionary scenarios proposed. Previous studies of the $\mathrm{CNO}$ abundances support production of the RCBs and the EHes by the DD scenario (Pandey et al. 2006). A dramatic advance was made by Clayton et al.'s (2005, 2007) discovery from mediumresolution infrared spectra of the $\mathrm{CO} 2.3 \mu \mathrm{m}$ bands that ${ }^{18} \mathrm{O}$ was very abundant for some cool RCBs and HdCs. Exploratory calculations led Clayton et al. (2007) to propose that ${ }^{18} \mathrm{O}$ was synthesized from ${ }^{14} \mathrm{~N}$ during the merger in the DD scenario. Extraordinary conditions are required for the FF scenario to lead to abundant ${ }^{18} \mathrm{O}$.

More recently, we have analyzed high-resolution $(R=$ $50,000)$ spectra of a few narrow windows in the $K$ band of the five known HdC stars and a few RCB stars (García-Hernández et al. 2009). In these spectra, the CO spectrum is resolved and application of spectrum synthesis enables a more precise estimate of the ${ }^{16} \mathrm{O} /{ }^{18} \mathrm{O}$ ratio to be obtained. Our recent analysis generally confirmed reports by Clayton and colleagues from $R=5900$ (or lower resolution) spectra. We confirm that the ${ }^{16} \mathrm{O} /{ }^{18} \mathrm{O}$ ratio is less than unity for those $\mathrm{HdC}$ stars (three of the known five) exhibiting $\mathrm{CO}$ lines in their spectra. However, ${ }^{16} \mathrm{O} /{ }^{18} \mathrm{O}=16$ was obtained for the cool RCB star S Aps; the other RCB stars in our sample were too warm to display $\mathrm{CO}$ molecular lines in their spectra. Our result ${ }^{16} \mathrm{O} /{ }^{18} \mathrm{O}=16$ for $\mathrm{S}$ Aps contrasts with Clayton et al.'s ratio of 4 . Our spectra show the gain in information resulting from the ability at the higher resolution to distinguish clearly different $\mathrm{CO}$ isomers. High-resolution spectra are essential to derive reliable and precise oxygen isotopic ratios for these hydrogen-deficient stars.

With S Aps showing a nearly 50-fold difference in the ${ }^{16} \mathrm{O} /{ }^{18} \mathrm{O}$ ratio from the analyzable $\mathrm{HdC}$ stars, the question arises: is a higher ratio characteristic of the RCBs? If so, this presumably provides a clue to understand the evolutionary relationship between $\mathrm{HdC}$ and RCB stars. Unfortunately, no new information may be drawn about the oxygen isotopic ratio in HdC stars until more HdC stars are discovered, but there are several RCBs with $\mathrm{CO}$ bands of a suitable strength not yet observed at high resolution. The example of $\mathrm{S}$ Aps shows that RCBs should be reobserved at higher resolution before drawing conclusions about the HdC-RCB connection. Therefore, we have extended high-resolution $K$-band observations to another five cool RCB stars in order to establish the range of the ${ }^{16} \mathrm{O} /{ }^{18} \mathrm{O}$ ratio among $\mathrm{RCBs}$ (Clayton et al.'s range from the six RCBs was 1 to $\geqslant 12$ ). Section 2 describes the high-resolution infrared spectroscopic observations and gives an overview of the observed spectra. Our abundance analysis and the results obtained are presented in Sections 3 and 4, respectively. The derived oxygen isotopic ratios are discussed in the context of the HdC-RCB connection in Section 5. Final conclusions are presented in Section 6. 


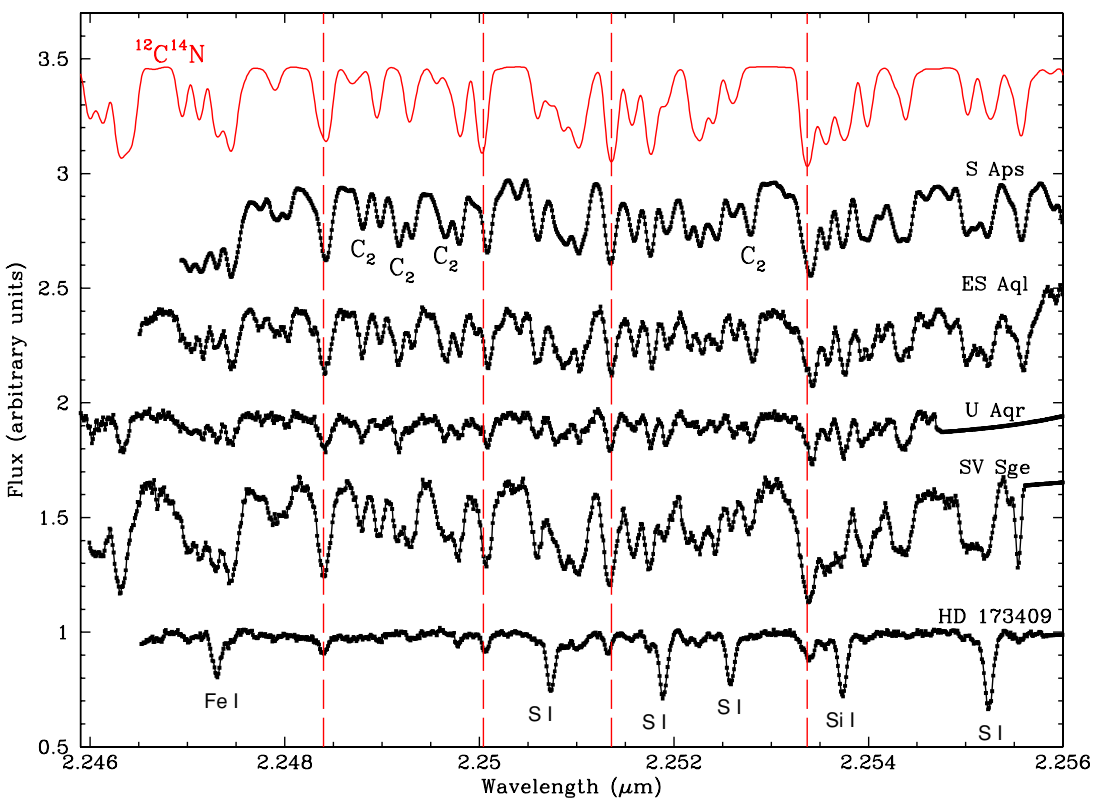

Figure 1. $K$-band spectra centered at $\sim 2.251 \mu \mathrm{m}$ for four cool RCBs (from top to bottom: S Aps, ES Aql, U Aqr, and SV Sge, respectively). The spectrum of the HdC star HD 173409 is also shown for comparison. Positions of several atomic lines are labeled. The strongest ${ }^{12} \mathrm{C}^{14} \mathrm{~N}$ features are indicated with a dashed red vertical line. $\mathrm{A}^{12} \mathrm{C}^{14} \mathrm{~N}$ synthetic spectrum (in red) composed for $\mathrm{S} A \mathrm{Aps}$ is also shown. Phillips system $\mathrm{C}_{2}$ lines labeled are 1-3 R22, 0-2 Q34, 1-3 Q8, and 1-3 Q10 in order of increasing wavelength.

(A color version of this figure is available in the online journal.)

\section{OBSERVATIONS AND $K$-BAND SPECTRA}

Our new sample contains six cool $\left(T_{\text {eff }} \leqslant 6250 \mathrm{~K}\right) \mathrm{RCB}$ stars plus one $\mathrm{HdC}$ star. Five of these stars are observed for the first time at high resolution. The RCB star S Aps and the HdC star HD 173409 were observed in spectral regions not previously covered by us (García-Hernández et al. 2009). High-resolution $(R \sim 50,000)$ spectroscopic observations were obtained in the period 2008 February-May with the PHOENIX spectrograph at Gemini South (Hinkle et al. 2003). We used the 0.34 arcsec slit at grating tilts centered at 2.251, 2.333, 2.349, and $2.365 \mu \mathrm{m}$. Each one of these wavelength settings provides a spectral coverage of $\sim 0.01 \mu \mathrm{m}$ (or $19.5 \mathrm{~cm}^{-1}$ ). Exposure times ranging from $\sim 30 \mathrm{~s}(K=5.4)$ to $\sim 10$ minutes $(K=8.3)$ per wavelength band were needed in order to reach a high signal-to-noise ratio ( $>100$ at the continuum). The spectral regions covered for each star in our sample are listed in Table 1. The observed spectra were reduced to one dimension by using standard tasks in $\mathrm{IRAF}^{7}$ and the telluric features were removed with the help of a spectrum of a hot star observed the same night. Standard air wavelengths are given hereafter.

Before attributing an observed spectrum to the stellar photosphere, we first determine if a star displays a significant infrared excess at $2.3 \mu \mathrm{m}$. For this purpose, we constructed spectral energy distributions of the stars in our sample by using the BVRIJHKL photometry available in the literature and the IRAS flux density at $12 \mu \mathrm{m}$. In the $K$ band, the flux from RCB stars SV Sge, S Aps, ${ }^{8}$ WX CrA, and U Aqr is predominantly from the photosphere; an infrared excess from circumstellar dust does not significantly contaminate our infrared spectra. The photospheric spectrum is also dominant for the HdC star HD 173409 with an effective temperature of $T_{\text {eff }}=6100 \mathrm{~K}$. Spectra of

\footnotetext{
7 IRAF is distributed by the National Optical Astronomy Observatory, which is operated by the Association of Universities for Research in Astronomy, Inc., under cooperative agreement with the National Science Foundation.

8 According to ASAS All Star Catalog (ASAS-3) observations, S Aps was recovering from a weak minimum during our observations.
}

$\mathrm{V}$ CrA and ES Aql are contaminated by infrared emission from dust.

Inspection of the light curve provided by the American Association of Variable Star Observers (AAVSO) ${ }^{9}$ and the ASAS All Star Catalog (ASAS- 3$)^{10}$ shows that V CrA experienced a decline of at least 6 mag beginning last 2007 March. AAVSO measurements are not reported for the period of our observations but the ASAS-3 measurements confirm that V CrA was at minimum during our observations. Certainly, our infrared spectra are featureless. $\mathrm{CO}$ and $\mathrm{CN}$ lines are expected to be quite strong in V CrA's photospheric spectrum $\left(T_{\text {eff }}=6250 \mathrm{~K}\right.$; Asplund et al. 2000 ) and, therefore, the observed featureless spectrum is most likely from the circumstellar dust.

ES Aql experienced a strong decline in brightness beginning in 2007 November. Fortunately, ES Aql was recovering from minimum light when observed by us; it was about 1 mag below maximum light according to AAVSO and ASAS-3 observations. Although ES Aql's photospheric spectrum may be partially diluted by emission from circumstellar dust, $\mathrm{CO}$ and $\mathrm{CN}$ lines are clearly detected in this star with $T_{\text {eff }}=5000 \mathrm{~K}$.

The observed spectral regions were chosen primarily to catch a mix of ${ }^{12} \mathrm{C}^{16} \mathrm{O},{ }^{12} \mathrm{C}^{18} \mathrm{O}$, as well as ${ }^{12} \mathrm{C}^{14} \mathrm{~N}$ lines. The region centered at $2.251 \mu \mathrm{m}$ was selected in order to be able to estimate the $\mathrm{N}$ abundance from $\mathrm{CN}$ lines. The CN molecule (Red System) contributes in all observed regions but this region is free from contributions from the first-overtone $\mathrm{CO}$ bands. In addition, a few lines of the $C_{2}$ Phillips bands $0-2$ and 1-3 together with some $\mathrm{S}$ I lines cross this interval. Figure 1 shows the observed spectra around $2.251 \mu \mathrm{m}$ for the stars in our sample.

The region centered at $2.333 \mu \mathrm{m}$ provides a selection of $\mathrm{P}$ and $\mathrm{R}$ lines from the ${ }^{12} \mathrm{C}^{16} \mathrm{O} 2-0$ and $3-1$ bands as well as a few weak ${ }^{12} \mathrm{C}^{14} \mathrm{~N}$ lines and the Na I line at $2.3348 \mu \mathrm{m}$. The isomer ${ }^{12} \mathrm{C}^{18} \mathrm{O}$ is not a contributor to this region. The observed spectra in the latter region are shown in Figure 2.

\footnotetext{
9 See http://www.aavso.org/

${ }^{10} \mathrm{See}$ http://www.astrouw.edu.pl/asas/?page=aasc
} 


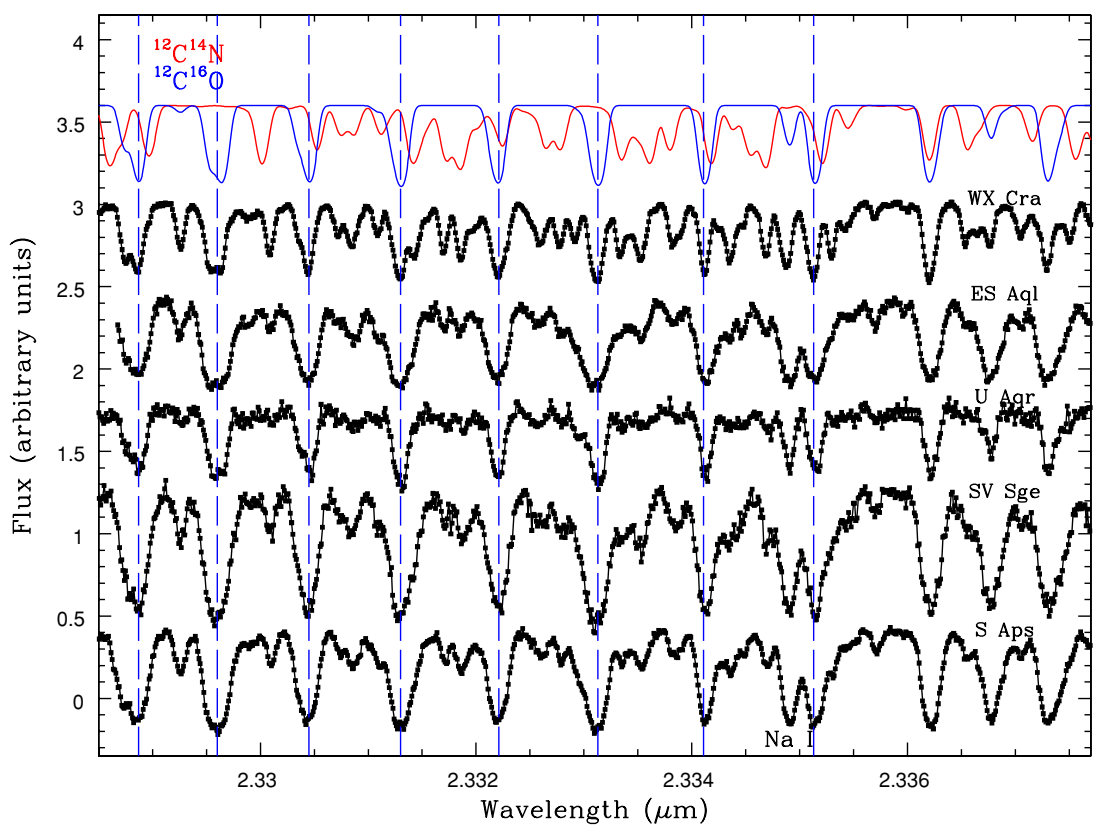

Figure 2. $K$-band spectra centered at $2.333 \mu \mathrm{m}$ for the five cool RCB stars in our sample. This spectral region is dominated by lines of the ${ }^{12} \mathrm{C}^{16} \mathrm{O}$ and ${ }^{12} \mathrm{C}^{14} \mathrm{~N}$ molecules. Note the $\mathrm{Na}$ I line at $2.3348 \mu \mathrm{m}$ that is usually blended with molecular features. The strongest ${ }^{12} \mathrm{C}^{16} \mathrm{O}$ lines are marked with a dashed blue vertical line. Synthetic spectra composed for WX CrA for the $\mathrm{CO}$ and $\mathrm{CN}$ isomers $\left({ }^{12} \mathrm{C}^{16} \mathrm{O}\right.$ in blue and ${ }^{12} \mathrm{C}^{14} \mathrm{~N}$ in red) indicate that these molecules dominate the spectra.

(A color version of this figure is available in the online journal.)

Table 1

Observation Summary of the Near-IR PHOENIX Observations ${ }^{\mathrm{a}}$

\begin{tabular}{lcc}
\hline \hline \multicolumn{1}{c}{ Star } & $\begin{array}{c}\text { Spectral Ranges Observed } \\
(\mu \mathrm{m})\end{array}$ & Date \\
\hline ES Aql & $2.251,2.332,2.349,2.366$ & 2008 Apr 8, 2008 Mar 21, 2008 May 13 \\
SV Sge & $2.251,2.332,2.366$ & 2008 May 13 \\
S Aps & $2.332,2.366$ & 2008 Feb 10 \\
WX CrA & $2.332,2.366$ & 2008 Apr 10, 2008 Mar 20 \\
U Aqr & $2.251,2.332,2.349,2.366$ & 2008 May 13 \\
V CrA & $2.251,2.332,2.349,2.366$ & 2008 Feb 29, 2008 Mar 20, 2008 Apr 7-10 \\
HD 173409 & 2.251 & 2008 Mar 21 \\
\hline
\end{tabular}

Note. ${ }^{\text {a }}$ The first six objects are cool RCB stars while HD 173409 is an HdC star.

Three stars were also observed in the interval around $2.349 \mu \mathrm{m}$. This region includes the $2-0{ }^{12} \mathrm{C}^{18} \mathrm{O}$ band head and also the $3-1{ }^{12} \mathrm{C}^{17} \mathrm{O}$ and $4-2{ }^{12} \mathrm{C}^{16} \mathrm{O}$ band heads at 2.351 and $2.352 \mu \mathrm{m}$, respectively. Figure 3 shows the $2.349 \mu \mathrm{m}$ ${ }^{12} \mathrm{C}^{18} \mathrm{O}$ 2-0 band of three cool RCB stars in our sample in comparison with the ${ }^{18} \mathrm{O}$-rich $\mathrm{HdC}$ star HD 137613 (García-Hernández et al. 2009). Note that strong ${ }^{12} \mathrm{C}^{18} \mathrm{O}$ lines are seen in the spectrum of the HdC star HD 137613, while these lines are weaker in the cool RCB stars U Aqr, ES Aql, and $\mathrm{S}$ Aps. Yet, the $4-2{ }^{12} \mathrm{C}^{16} \mathrm{O}$ band head (and other ${ }^{12} \mathrm{C}^{16} \mathrm{O}$ lines) is stronger in the RCB spectra than in the spectrum of HD 137613.

Finally, all stars were observed in the region centered at $2.365 \mu \mathrm{m}$. This region is well suited to providing an estimate of the ${ }^{16} \mathrm{O} /{ }^{18} \mathrm{O}$ ratio because it is longward of the $2-0{ }^{12} \mathrm{C}^{18} \mathrm{O} R$ branch head at $2.349 \mu \mathrm{m}$ and includes weak and strong ${ }^{12} \mathrm{C}^{16} \mathrm{O}$ and ${ }^{12} \mathrm{C}^{18} \mathrm{O}$ lines that are cleanly separated at $R=50,000$. There are also ${ }^{12} \mathrm{C}^{14} \mathrm{~N}$ lines in this region. Figure 4 shows the observed spectra around $2.365 \mu \mathrm{m}$ for the cool RCB stars in our sample. It is clear that the RCB star WX CrA is strongly enriched in ${ }^{18} \mathrm{O}$ because lines of ${ }^{12} \mathrm{C}^{16} \mathrm{O}$ and ${ }^{12} \mathrm{C}^{18} \mathrm{O}$ are similar in strength, as anticipated from medium-resolution spectra (Clayton et al. 2007). In contrast, the ${ }^{12} \mathrm{C}^{18} \mathrm{O}$ lines-especially the cleanest ${ }^{12} \mathrm{C}^{18} \mathrm{O}$ line at $\sim 2.3686 \mu \mathrm{m}$ - are much weaker in the other four RCB stars, suggesting that ${ }^{18} \mathrm{O}$ is less abundant in these stars.

\section{ABUNDANCE ANALYSIS}

We derive oxygen isotopic abundances by using spectral synthesis techniques. A detailed description of the adopted abundance analysis procedure as well as the input physics and atomic and molecular line lists has been presented by us (García-Hernández et al. 2009) and will not be repeated here. In short, we have constructed H-deficient spherically symmetric MARCS model atmospheres for the stars in our sample and we considered up-to-date lists of atomic and molecular lines. The most important contributors to lines in our spectral regions are the $\mathrm{CO}$ and $\mathrm{CN}$ molecules. We consider line lists for all the $\mathrm{CO}$ and $\mathrm{CN}$ isomers (i.e., ${ }^{12} \mathrm{C}^{16} \mathrm{O},{ }^{12} \mathrm{C}^{17} \mathrm{O},{ }^{12} \mathrm{C}^{18} \mathrm{O},{ }^{12} \mathrm{C}^{14} \mathrm{~N},{ }^{12} \mathrm{C}^{15} \mathrm{~N}$, etc.).

The MARCS models follow the prescription discussed by Asplund et al. (1997), where the input chemical composition is that of the RCB stars (i.e., $\log \epsilon(\mathrm{H})=7.5$ and a $\mathrm{C} / \mathrm{He}$ ratio of $1 \%$ by number density ${ }^{11}$; Rao \& Lambert 1994). The only exception

\footnotetext{
11 The abundances are normalized to $\log \sum \mu_{i} \varepsilon_{i}=12.15$, where $\mu_{i}$ is the mean atomic weight of element $i$, i.e., $\log \varepsilon(\mathrm{He})=11.52$ and $\log \varepsilon(\mathrm{C})=9.52$; see Asplund et al. (1997).
} 


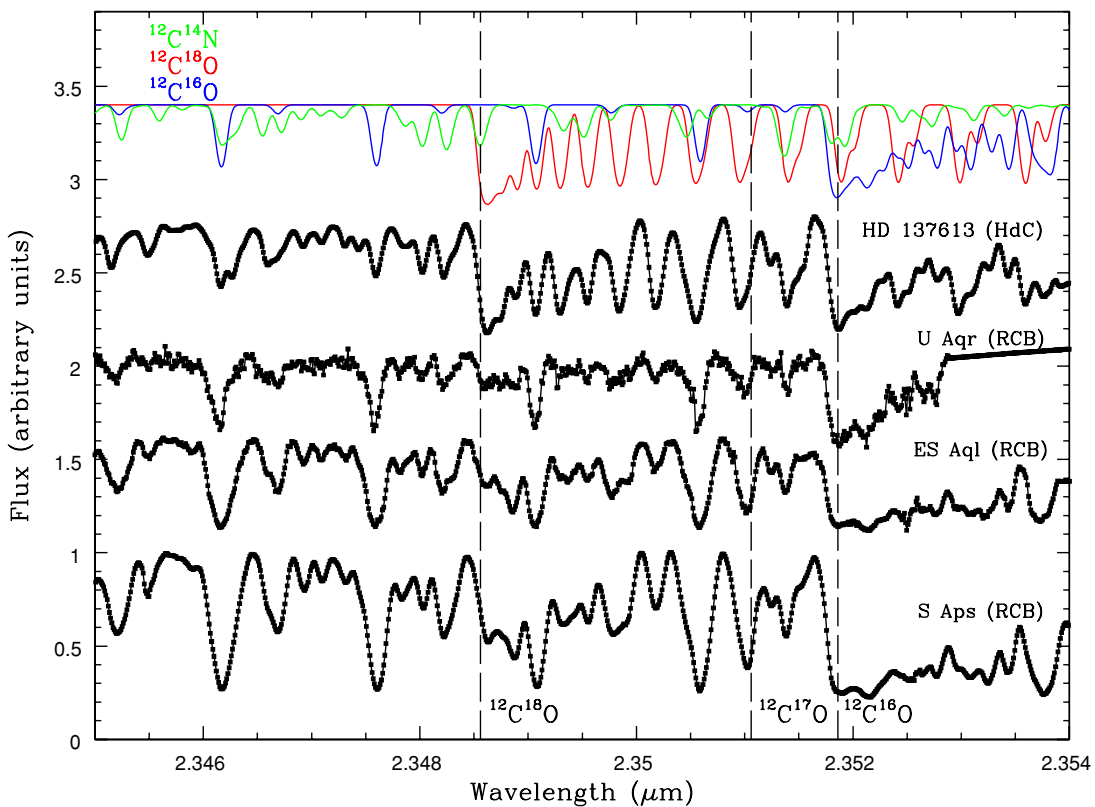

Figure 3. $K$-band spectra centered at $\sim 2.349 \mu \mathrm{m}$ for three cool RCB stars (U Aqr, ES Aql, and S Aps) and the ${ }^{18} \mathrm{O}$-rich $\mathrm{HdC}$ star HD 137613 (taken from García-Hernández et al. 2009). Wavelengths of the $2-0{ }^{12} \mathrm{C}^{18} \mathrm{O}, 3-1{ }^{12} \mathrm{C}^{17} \mathrm{O}$, and $4-2{ }^{12} \mathrm{C}^{16} \mathrm{O}$ band heads are marked with a vertical dashed line. Synthetic spectra composed for HD 137613 are shown for the isomers ${ }^{12} \mathrm{C}^{16} \mathrm{O}$ in blue, ${ }^{12} \mathrm{C}^{18} \mathrm{O}$ in red, and ${ }^{12} \mathrm{C}^{14} \mathrm{~N}$ in green.

(A color version of this figure is available in the online journal.)

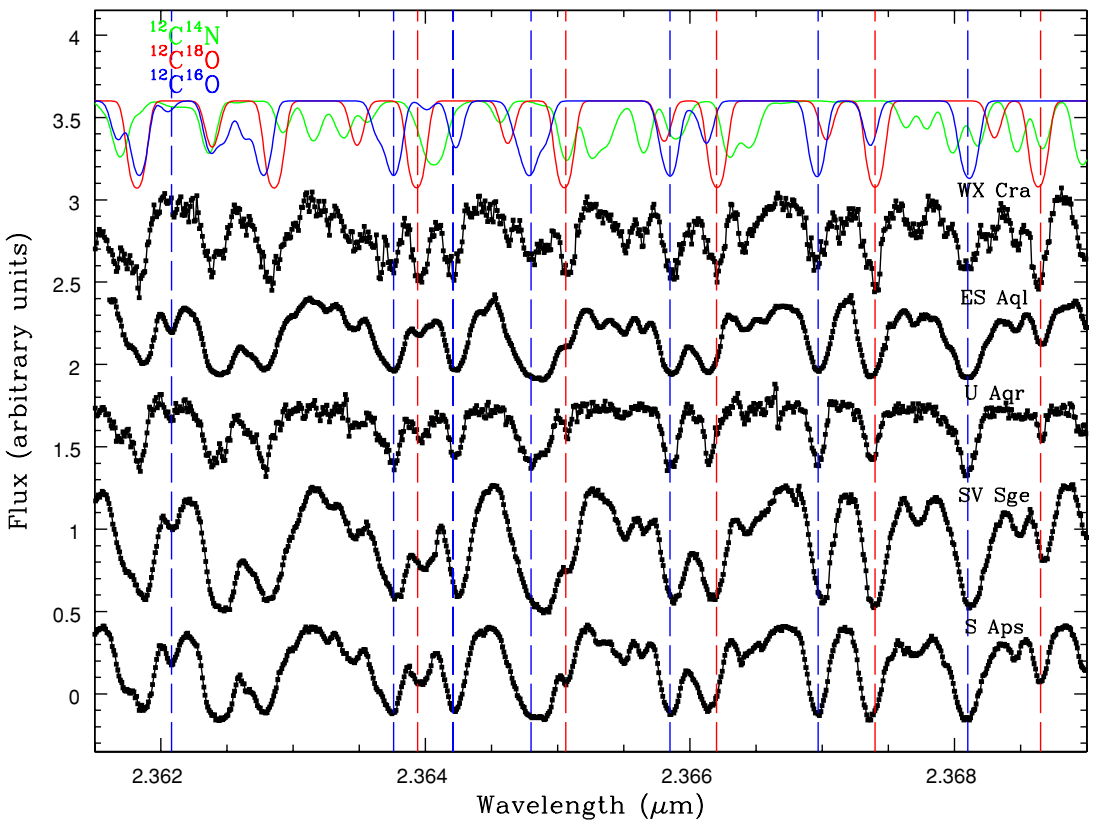

Figure 4. $K$-band spectra centered at $2.365 \mu \mathrm{m}$ of the five cool RCB stars in our sample. Synthetic spectra composed for WX Cra for the CO isomers $\left({ }^{12} \mathrm{C}^{16} \mathrm{O}\right.$ in blue and ${ }^{12} \mathrm{C}^{18} \mathrm{O}$ in red) and for the ${ }^{12} \mathrm{C}^{14} \mathrm{~N}$ isomer (in green) are shown at the top for comparison. Some lines of ${ }^{12} \mathrm{C}^{18} \mathrm{O}$ and ${ }^{12} \mathrm{C}^{16} \mathrm{O}$ are resolved and marked with dashed vertical lines. Note that the strongest ${ }^{12} \mathrm{C}^{16} \mathrm{O}$ and ${ }^{12} \mathrm{C}^{18} \mathrm{O}$ lines are saturated in the RCB stars: ES Aql, SV Sge, and S Aps. However, weaker and clean ${ }^{12} \mathrm{C}^{18} \mathrm{O}$ and ${ }^{12} \mathrm{C}^{16} \mathrm{O}$ lines such as those at $\sim 2.3686 \mu \mathrm{m}$ and $\sim 2.3620 \mu \mathrm{m}$ indicate that ${ }^{18} \mathrm{O}$ is less abundant in these three RCBs compared with the ${ }^{18} \mathrm{O}-$ rich RCB WX CrA.

(A color version of this figure is available in the online journal.)

with respect to initial composition was U Aqr (see below) for which we have also used a metal-poor model with $[\mathrm{Fe} / \mathrm{H}]=$ -1.0 dex. The TURBOSPECTRUM package (Alvarez \& Plez 1998), which is compatible with MARCS, is used to generate the corresponding synthetic spectra.

Apart from the adopted input composition, a microturbulent velocity, surface gravity, and effective temperature must be chosen. The warm RCB stars display an average micro- turbulent velocity of $6.6 \mathrm{~km} \mathrm{~s}^{-1}$ with no apparent trend with effective temperature (Asplund et al. 2000). Thus, we assumed a microturbulence of $7 \mathrm{~km} \mathrm{~s}^{-1}$ for all RCB stars. This value provides a fair fit to both the weaker and stronger lines of a given species, a necessary condition according to the definition of microturbulence. When a synthetic spectrum computed for a microturbulence of $7 \mathrm{~km} \mathrm{~s}^{-1}$ is convolved with a Gaussian profile with a width of $6 \mathrm{~km} \mathrm{~s}^{-1}$ in order to take into account the 
Table 2

Derived Chemical Abundances in Cool RCB Stars ${ }^{\mathrm{a}}$

\begin{tabular}{|c|c|c|c|c|c|c|c|c|}
\hline Star & $T_{\text {eff }}(\mathrm{K})^{\mathrm{b}}$ & ${ }^{16} \mathrm{O} /{ }^{18} \mathrm{O}^{\mathrm{c}}$ & ${ }^{16} \mathrm{O} /{ }^{18} \mathrm{O}$ & ${ }^{16} \mathrm{O} /{ }^{17} \mathrm{O}$ & ${ }^{14} \mathrm{~N} /{ }^{15} \mathrm{~N}$ & $\mathrm{C} / \mathrm{N} / \mathrm{O}^{\mathrm{d}}$ & $\mathrm{Na}$ & $\langle S\rangle$ \\
\hline SV Sge & 4000 & 4 & $16 \pm 4$ & $>20$ & $>10$ & $9.5 / 7.7 / 8.4$ & $\cdots$ & 7.6 \\
\hline S Aps & 5400 & 4 & $16 \pm 4$ & $>80$ & $>10$ & $9.5 / 9.2 / 9.2$ & $\ldots$ & 6.8 \\
\hline WX CrA & 5300 & 1 & $0.3 \pm 0.1$ & $>6$ & $\cdots$ & $9.5 / 9.3 / 8.7$ & 7.3 & $\ldots$ \\
\hline V CrA ${ }^{f}$ & 6250 & $\ldots$ & $\ldots$ & $\ldots$ & $\ldots$ & $\ldots$ & $\ldots$ & $\ldots$ \\
\hline HD 173409 & 6100 & $\cdots$ & $\cdots$ & $\cdots$ & $>4$ & $9.5 / 9.2 / 8.7$ & 7.0 & 7.6 \\
\hline
\end{tabular}

Notes.

a The abundances are normalized to $\log \sum \mu_{i} \epsilon_{i}=12.15$, i.e., $\log \varepsilon(\mathrm{He})=11.52$ and $\log \varepsilon(\mathrm{C})=9.52$. The error in the derived

${ }^{16} \mathrm{O} /{ }^{18} \mathrm{O}$ ratios is estimated from the typical uncertainty of \pm 0.05 dex associated with the corresponding ${ }^{16} \mathrm{O}$ and ${ }^{18} \mathrm{O}$ isotopic abundances.

b References. Clayton et al. 2007; Asplund et al. 1997, 1998; Lawson et al. 1990.

${ }^{c}{ }^{16} \mathrm{O} /{ }^{18} \mathrm{O}$ ratios derived from medium-resolution near-IR spectra by Clayton et al. (2007).

$\mathrm{d}$ The CNO abundances are given for the assumed input composition, i.e., $\log \varepsilon(\mathrm{Fe})=7.2, \log \varepsilon(\mathrm{Na})=6.8, \log \varepsilon(\mathrm{S})=7.5$, etc. Asplund et al. (1997).

e The $K$-band spectrum is significantly obscured by circumstellar dust. Note that the CNO abundances from the simultaneous spectra taken on 2008 April 8 are given.

f The $K$-band spectrum is largely from circumstellar dust, and the photospheric spectrum is greatly obscured or diluted.

instrumental profile at $R=50,000$, a good fit is obtained to the line profiles. This suggests that the macroturbulence is small. In addition, a surface gravity of $\log g=+0.5$ was assumed in the construction of the model atmospheres for all RCB stars. This is consistent with the gravities found by Asplund et al. (2000) in their optical spectroscopic analysis of the cooler $\left(T_{\text {eff }}<6500 \mathrm{~K}\right)$ RCB stars. Finally, for each star in our sample we chose the effective temperatures listed by Clayton et al. (2007) for their analysis of medium-resolution near-infrared spectra (see their Table 2). The adopted $T_{\text {eff }}$ are listed in Table 2 . It is to be noted that we do not precisely know the effective temperatures of our sample stars and they cannot be derived from our highresolution spectra. Note also that by selecting the Clayton et al's temperatures we can compare our derived oxygen isotopic ratios with those obtained from medium-resolution spectra. In any case, our main goal is to determine the ${ }^{16} \mathrm{O} /{ }^{18} \mathrm{O}$ oxygen isotopic ratios, and their determination is almost independent of the adopted model parameters, as shown in our previous paper and in Table 2 for SV Sge and U Aqr for which two different models are used for each star. According to our near-infrared and our previous optical spectra, SV Sge looks hotter than the $4000 \mathrm{~K}$ adopted by Clayton et al. U Aqr according to Bond et al. (1979) from their analysis of a few strong optical lines has a calcium abundance similar to that of HD 182040, i.e., the metallicity is approximately solar. (U Aqr is noted for its remarkably high abundances of light $s$-process abundances- $-\mathrm{Sr}$ and $\mathrm{Zr}$.) Clayton et al. (2007) adopted $T_{\text {eff }}=6000 \mathrm{~K}$ from Asplund et al. (1997). Cottrell \& Lawson (1998) suggest U Aqr belongs to the Galactic halo. Then, it might be metal-poor. There is spectroscopic evidence for this and for a lower effective temperature (Rao 2008). Thus, for U Aqr we consider a model with $T_{\text {eff }}=6000 \mathrm{~K}$ and a metal-poor $([\mathrm{Fe} / \mathrm{H}]=-1)$ model with $T_{\text {eff }}=5400 \mathrm{~K}$.

The corresponding synthetic spectra were compared to the observed ones in order to determine the isotopic ratios for $\mathrm{O}$ and $\mathrm{N}$-see García-Hernández et al. (2009) for a detailed description of the procedure employed in the derivation of isotopic abundances in hydrogen-deficient stars. We also list the derived $\mathrm{O}$ and $\mathrm{N}$ elemental abundances for the assumed input composition (i.e., $\log \varepsilon(\mathrm{H})=7.5, \log \varepsilon(\mathrm{He})=11.52$, and $\log \varepsilon(C)=9.52)$ together with, when possible, the abundances of the heavier elements $\mathrm{Na}$ and $\mathrm{S}$ from the $\mathrm{Na}$ I line at $2.3348 \mu \mathrm{m}^{12}$ and the few $\mathrm{S}$ I lines around $2.251 \mu \mathrm{m}$. It should be noted here that the listed $\mathrm{O}, \mathrm{N}, \mathrm{Na}$, and $\mathrm{S}$ elemental abundances must be considered with caution because they are strongly dependent on the atmospheric parameters (Table 2). The formal error in the total $\mathrm{N}$ and $\mathrm{O}$ abundances taking into account variations of the atmospheric parameters used in the modeling of hydrogen-deficient stars is estimated to be of the order of 0.3-0.4 dex (García-Hernández et al. 2009). In addition, the $\mathrm{C}_{2}$ Phillips system lines 0-2 Q(34), 1-3 Q(8), and 1-3 Q(10) (see Figure 1) observed in HdC and cool RCB stars exhibit a "carbon problem," which complicates their use for carbon abundance determinations (García-Hernández et al. 2009 and references therein). Thus, we have to assume the input $C$ abundance $(\log \varepsilon(C)=9.52)$ in our abundance analysis, which affects the derived $\mathrm{N}$ and $\mathrm{O}$ elemental abundances. The uncertainties for the derived $\mathrm{Na}$ and $\mathrm{S}$ abundances are even higher than those estimated for $\mathrm{N}$ and $\mathrm{O}$ with a typical value of $\sim 0.6-0.7$ dex - especially in these cool RCB stars where the atomic lines are usually blended with the strong $\mathrm{CN}$ and $\mathrm{CO}$ molecular features across the spectra.

\section{OXYGEN ISOTOPIC RATIOS}

For the RCB stars WX CrA, ES Aql, U Aqr, and SV Sge we are able for the first time to get reliable estimates of the ${ }^{16} \mathrm{O} /{ }^{18} \mathrm{O}$ isotopic ratio. The ${ }^{16} \mathrm{O} /{ }^{18} \mathrm{O}$ ratio of 16 obtained for the $\mathrm{RCB}$ star S Aps was previously known from spectrum synthesis at $2.349 \mu \mathrm{m}$ (García-Hernández et al. 2009). For the RCB star $\mathrm{V} \mathrm{CrA}$, the $K$-band spectra are featureless because they are dominated by emission from circumstellar dust (see Section 2) precluding a measurement of the ${ }^{16} \mathrm{O} /{ }^{18} \mathrm{O}$ ratio. For the five RCB stars, lower limits to the ${ }^{16} \mathrm{O} /{ }^{17} \mathrm{O}$ and ${ }^{14} \mathrm{~N} /{ }^{15} \mathrm{~N}$ ratios from the absence of detectable ${ }^{12} \mathrm{C}^{17} \mathrm{O}$ and ${ }^{12} \mathrm{C}^{15} \mathrm{~N}$ lines are also determined. Unfortunately, we can not give estimates of the

\footnotetext{
12 Note that the $2.3348 \mu \mathrm{m} \mathrm{Na}$ I line is strongly blended with molecular features, preventing us from measuring a reliable $\mathrm{Na}$ abundance in most of the sample stars.
} 


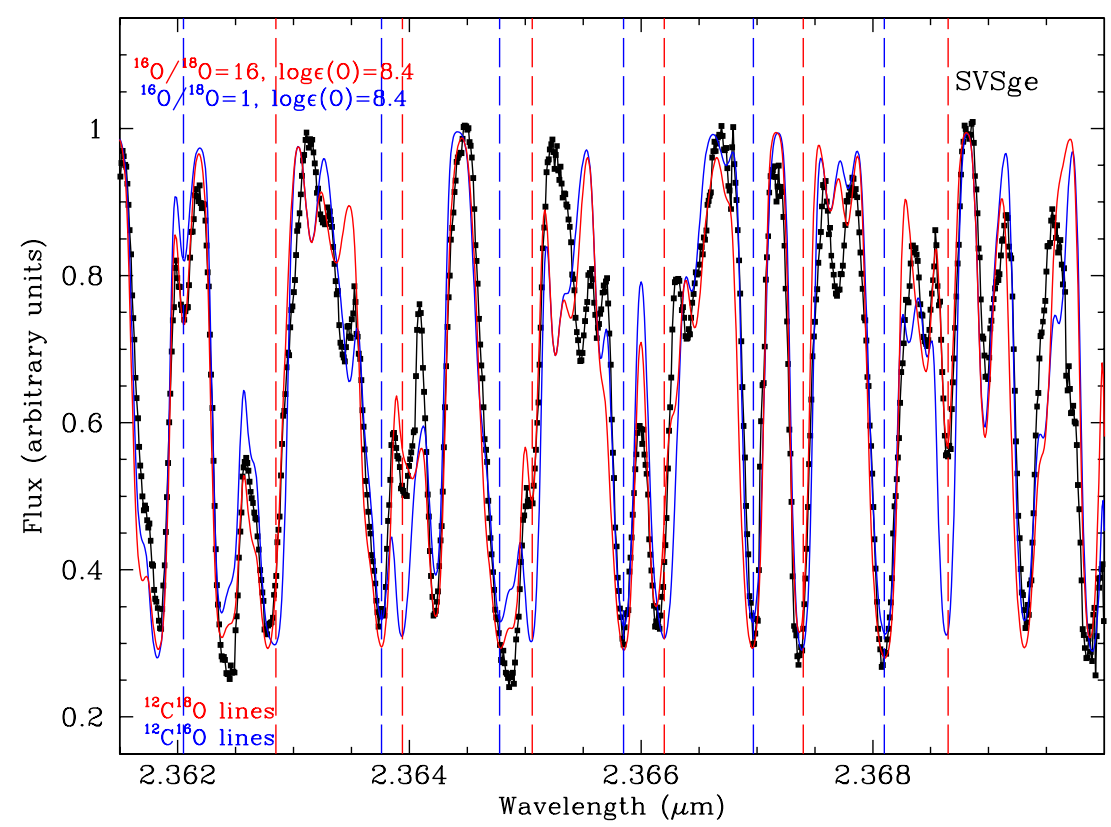

Figure 5. Best synthetic (red) and observed (black) spectra for the region centered at $2.365 \mu \mathrm{m}$ for the RCB star SV Sge. The red spectrum assumes $T_{\text {eff }}=4000 \mathrm{~K}$, the input $\mathrm{C}$ abundance of 9.52 , the $\mathrm{N}$ abundance of 7.7 , a total $\mathrm{O}\left({ }^{16} \mathrm{O}+{ }^{18} \mathrm{O}\right)$ of $\log \varepsilon(\mathrm{O})=8.4$, and the isotopic ratio ${ }^{16} \mathrm{O} /{ }^{18} \mathrm{O}=16$. Selected ${ }^{12} \mathrm{C}^{18} \mathrm{O}$ and ${ }^{12} \mathrm{C}^{16} \mathrm{O}$ lines are marked with red and blue vertical dashed lines, respectively. A synthetic spectrum (blue) for the same total $\mathrm{O}\left({ }^{16} \mathrm{O}+{ }^{18} \mathrm{O}\right)$ of $\log \varepsilon(\mathrm{O})=8.4$ but a lower ${ }^{16} \mathrm{O} /{ }^{18} \mathrm{O}$ ratio of 1 (i.e., a higher ${ }^{18} \mathrm{O}$ abundance) is shown for comparison. Note that the strongest ${ }^{12} \mathrm{C}^{16} \mathrm{O}$ and ${ }^{12} \mathrm{C}^{18} \mathrm{O}$ lines are saturated. The weaker and cleanest ${ }^{12} \mathrm{C}^{18} \mathrm{O}$ and ${ }^{12} \mathrm{C}^{16} \mathrm{O}$ lines such as those at $\sim 2.3686 \mu \mathrm{m}$ and $\sim 2.3620 \mu \mathrm{m}$, respectively, provide the ratio ${ }^{16} \mathrm{O} /{ }^{18} \mathrm{O}=16$

(A color version of this figure is available in the online journal.)

${ }^{12} \mathrm{C} /{ }^{13} \mathrm{C}$ ratios because our observations did not cover the $2-0$ ${ }^{13} \mathrm{C}^{16} \mathrm{O}$ band head at $2.344 \mu \mathrm{m}$. Individual ${ }^{13} \mathrm{C}^{16} \mathrm{O}$ features are not detectable, but this is not surprising given that previous albeit scanty evidence for RCBs is that they have a high ${ }^{12} \mathrm{C} /{ }^{13} \mathrm{C}$ ratio. Note that we observed that the HdC star HD 173409 around $2.251 \mu \mathrm{m}$ and a lower limit to the ${ }^{14} \mathrm{~N} /{ }^{15} \mathrm{~N}$ ratio as well as the $\mathrm{S}$ elemental abundance from the $\mathrm{S}$ I lines are also given. Table 2 summarizes the derived abundances.

By inspection of Table 2, the ${ }^{16} \mathrm{O} /{ }^{18} \mathrm{O}$ ratios (with the sole exception of WX CrA) of these RCB stars differ from those reported by Clayton et al. (2007) from medium-resolution spectra. Clayton et al. (2007) underestimated the ${ }^{16} \mathrm{O} /{ }^{18} \mathrm{O}$ ratios for the RCB stars ES Aql, S Aps, and SV Sge. This may be because most of the ${ }^{12} \mathrm{C}^{16} \mathrm{O}$ lines in these three stars are saturated, not being useful for abundance analysis - we derive the isotopic ${ }^{16} \mathrm{O}$ and ${ }^{18} \mathrm{O}$ abundances from the weaker and cleanest lines in the observed spectra around 2.333 and $2.365 \mu \mathrm{m}$. This is illustrated in Figure 5 for the RCB star SV Sge. The ${ }^{16} \mathrm{O} /{ }^{18} \mathrm{O}$ ratio of 16 is obtained in this star for which Clayton et al. obtained a value of 4. For those stars (e.g., ES Aql, U Aqr, and S Aps) with available spectra in the 2.349 and $2.365 \mu \mathrm{m}$ regions, the derived ${ }^{16} \mathrm{O} /{ }^{18} \mathrm{O}$ ratios from both regions are in excellent agreement. For the RCB star U Aqr, our ${ }^{16} \mathrm{O} /{ }^{18} \mathrm{O}$ estimate of 4 contrasts with the lower limit of 12 estimated by Clayton et al. Synthetic spectra for U Aqr around 2.333 and $2.365 \mu \mathrm{m}$ are shown in Figures 6 and 7, respectively. The ${ }^{16} \mathrm{O} /{ }^{18} \mathrm{O}$ ratio of 20 displayed by ES Aql contrasts with Clayton et al.'s estimate of 6 for this star. Figures 8 and 9 show synthetic spectra for this star around the $2-0{ }^{12} \mathrm{C}^{18} \mathrm{O}$ band head at $2.349 \mu \mathrm{m}$ and around $2.365 \mu \mathrm{m}$, respectively. It is to be noted that the ${ }^{12} \mathrm{C}^{16} \mathrm{O}$ lines are saturated for this star with $T_{\text {eff }}=5000 \mathrm{~K}$. In addition, the $K$-band spectra for ES Aql are partially diluted by circumstellar dust with dilution decreasing between 2008 March and May. This is deduced from the fact that synthetic spectra predict stronger ${ }^{12} \mathrm{C}^{16} \mathrm{O}$ lines than observed and the CO lines are stronger in the 2008 May spectrum than in 2008 April. ASAS-3 visual observations show that the star has brightened by about 1 mag between March and May when it was about $0.5 \mathrm{mag}$ below maximum light. Although the derived ${ }^{16} \mathrm{O}$ and ${ }^{18} \mathrm{O}$ isotopic abundances differ by about $0.2 \mathrm{dex}$ from analyses of the 2008 March 21 and April 8 spectra, the ${ }^{16} \mathrm{O} /{ }^{18} \mathrm{O}$ ratio of 20 remains unchanged (Figures 8 and 9). Finally, the RCB star WX CrA seems to be an HdC-like $\left({ }^{18} \mathrm{O}\right.$-rich) star with ${ }^{16} \mathrm{O} /{ }^{18} \mathrm{O}=0.3$, where a value of 1 was provided by Clayton and colleagues. Synthetic and observed spectra around $2.365 \mu \mathrm{m}$ for WX CrA are shown in Figure 10.

The ${ }^{16} \mathrm{O} /{ }^{18} \mathrm{O}$ ratios derived from high-resolution spectra are in fair agreement with those derived from medium-resolution spectra when the molecular lines of the different $\mathrm{CO}$ isomers are not strongly saturated. This is the case for the ${ }^{18} \mathrm{O}$-rich $\mathrm{HdC}$ stars (García-Hernández et al. 2009) and the RCB star WX CrA. In short, high-resolution spectra are needed in order to derive reliable oxygen isotopic ratios in cool RCB stars with strong $\mathrm{CO}$ molecular lines.

In summary, there is a clear difference between the ${ }^{16} \mathrm{O} /{ }^{18} \mathrm{O}$ ratios of the $\mathrm{HdC}$ and $\mathrm{RCBs}$ for those stars for which a measurement is possible. This difference suggested by interpretation of medium-resolution spectra is confirmed and extended by our high-resolution spectra. All (three) HdCs with $\mathrm{CO}$ lines show that ${ }^{18} \mathrm{O}$ is more abundant than ${ }^{16} \mathrm{O}$. All (with the only exception of WX CrA) of the investigated RCBs show that ${ }^{16} \mathrm{O}$ is more abundant than ${ }^{18} \mathrm{O} .{ }^{13}$

\section{THE HdC-RCB CONNECTION}

Given the extreme rarity of $\mathrm{HdC}$ and $\mathrm{RCB}$ stars, it seems reasonable to suppose that they are closely related. Here, we

\footnotetext{
${ }^{13}$ The cool RCB Z UMi has ${ }^{16} \mathrm{O} /{ }^{18} \mathrm{O} \geqslant 8$ according to Clayton et al. (2007).
} 


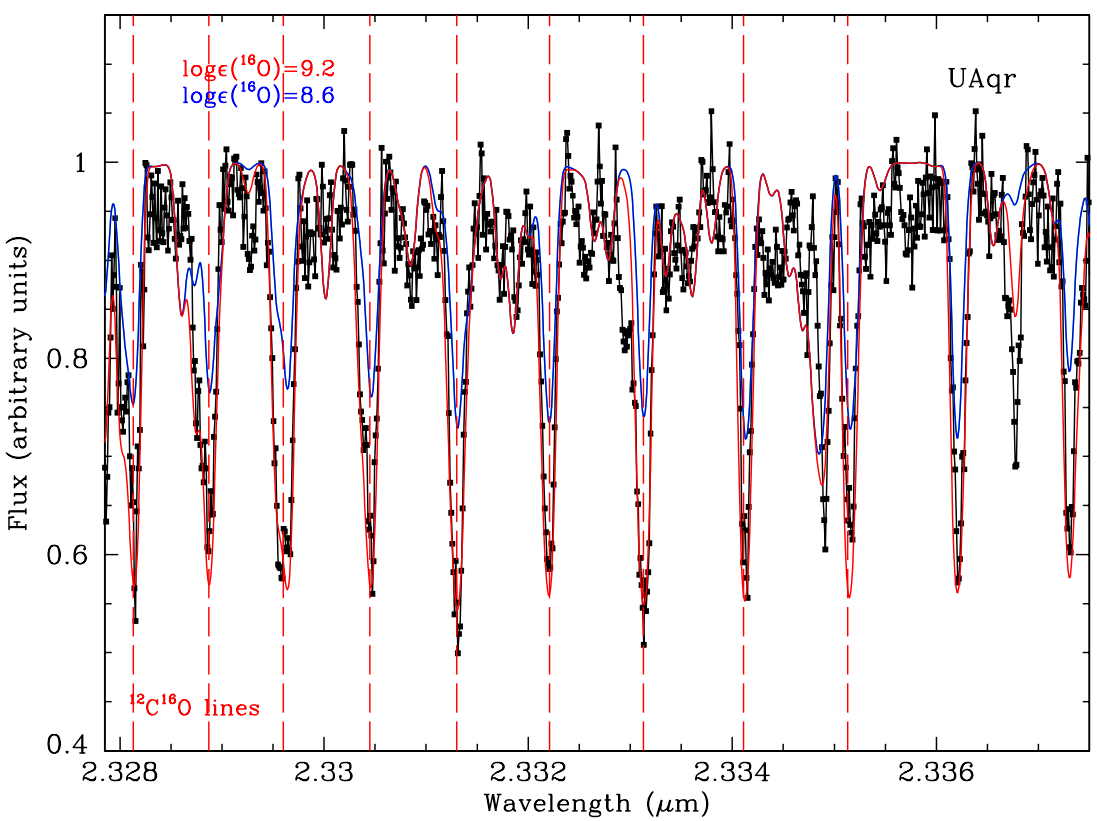

Figure 6. Best synthetic (red) and observed (black) spectra for the wavelength region centered around $2.333 \mu \mathrm{m}$ for the RCB star U Aqr. The red spectrum assumes $T_{\text {eff }}=6000 \mathrm{~K}$, the input $\mathrm{C}$ abundance of 9.52 , the $\mathrm{N}$ abundance of 9.4 , and the ${ }^{16} \mathrm{O}$ isotopic abundance of $\log \varepsilon\left({ }^{16} \mathrm{O}\right)=9.2$. This region contains ${ }^{12} \mathrm{C}^{16} \mathrm{O}$ but not ${ }^{12} \mathrm{C}^{18} \mathrm{O}$ lines, and the ${ }^{12} \mathrm{C}^{16} \mathrm{O}$ lines are fitted with $\log \varepsilon\left({ }^{16} \mathrm{O}\right)=9.2$. The synthetic spectrum also includes $\mathrm{CN}$ lines. A synthetic spectrum (blue) for a lower ${ }^{16} \mathrm{O}$ abundance of $\log \varepsilon\left({ }^{16} \mathrm{O}\right)=8.6$ is shown for comparison. Note the strong dependence of the ${ }^{12} \mathrm{C}^{16} \mathrm{O}$ lines (marked with red dashed vertical lines) to changes of the ${ }^{16} \mathrm{O}$ abundance.

(A color version of this figure is available in the online journal.)

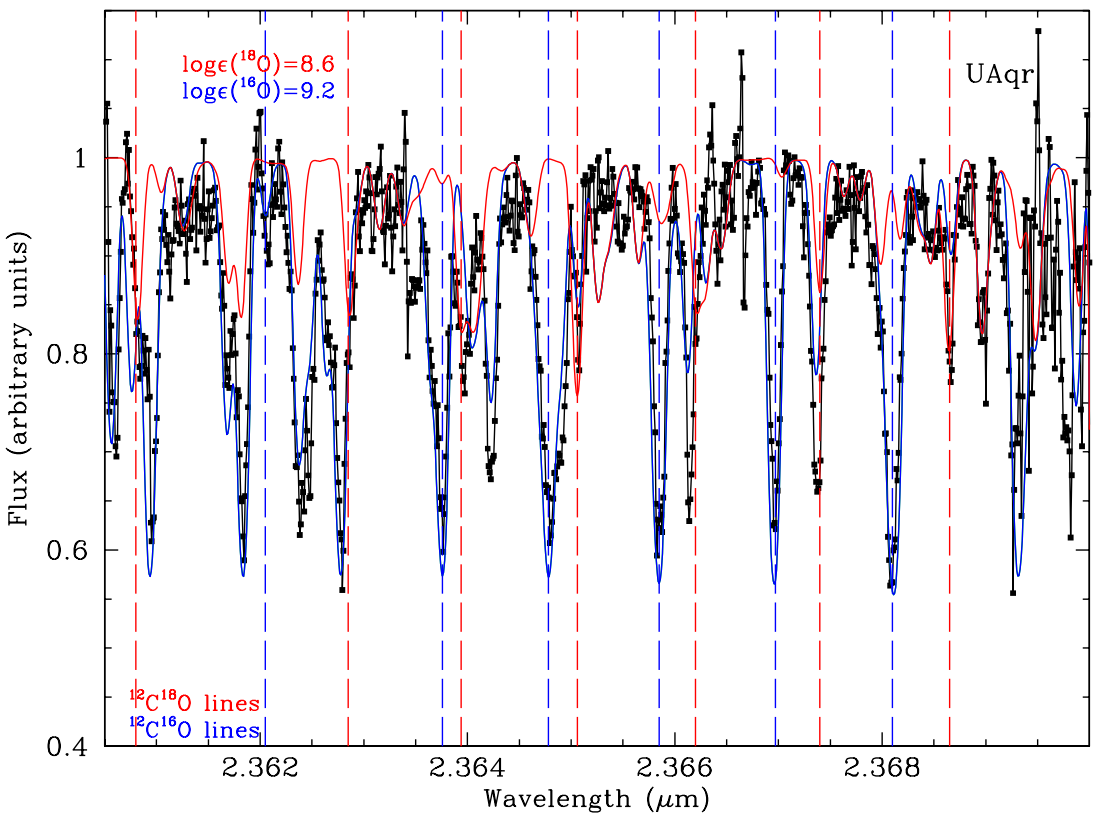

Figure 7. Synthetic spectra for the different ${ }^{12} \mathrm{C}^{16} \mathrm{O}$ and ${ }^{12} \mathrm{C}^{18} \mathrm{O}$ isomers (blue and red, respectively) and observed (black) spectrum in the wavelength region centered around $2.365 \mu \mathrm{m}$ for the RCB star U Aqr. Note that the synthetic spectra also include $\mathrm{CN}$ lines. The synthetic spectra are constructed by assuming $T_{\text {eff }}=6000 \mathrm{~K}$, the input $\mathrm{C}$ abundance of 9.52 , and using the $\mathrm{N}$ abundance of 9.4 . The blue and red synthetic spectra correspond to the oxygen isotopic abundances of log $\varepsilon\left({ }^{16} \mathrm{O}\right)=9.2$ and $\log \varepsilon\left({ }^{18} \mathrm{O}\right)=8.6$, respectively (or the isotopic ratio ${ }^{16} \mathrm{O} /{ }^{18} \mathrm{O}=4$ ).

(A color version of this figure is available in the online journal.)

adopt the view that both are products of the DD scenario in which a $\mathrm{He}$ WD merges with a $\mathrm{C}-\mathrm{O}$ WD. The merger inflates the resultant atmosphere around the $\mathrm{C}-\mathrm{O} \mathrm{WD}$ to supergiant dimensions. A hot H-deficient supergiant is created that is expected to evolve rapidly to lower temperatures at approximately constant luminosity. The star is a cool supergiant for about $10^{5}-10^{6}$ years before the onset of rapid evolution back to high temperatures and a final descent of the WD cooling track. HdCs and RCBs are to be identified with the cool supergiant phase.

The precise form of the evolutionary track in the H-R diagram may depend on multiple aspects of the merger, e.g., the masses and compositions of the WDs, the rate of mass transfer from the $\mathrm{He} \mathrm{WD}$ to the $\mathrm{C}-\mathrm{O} \mathrm{WD}$, and the gravitational and 


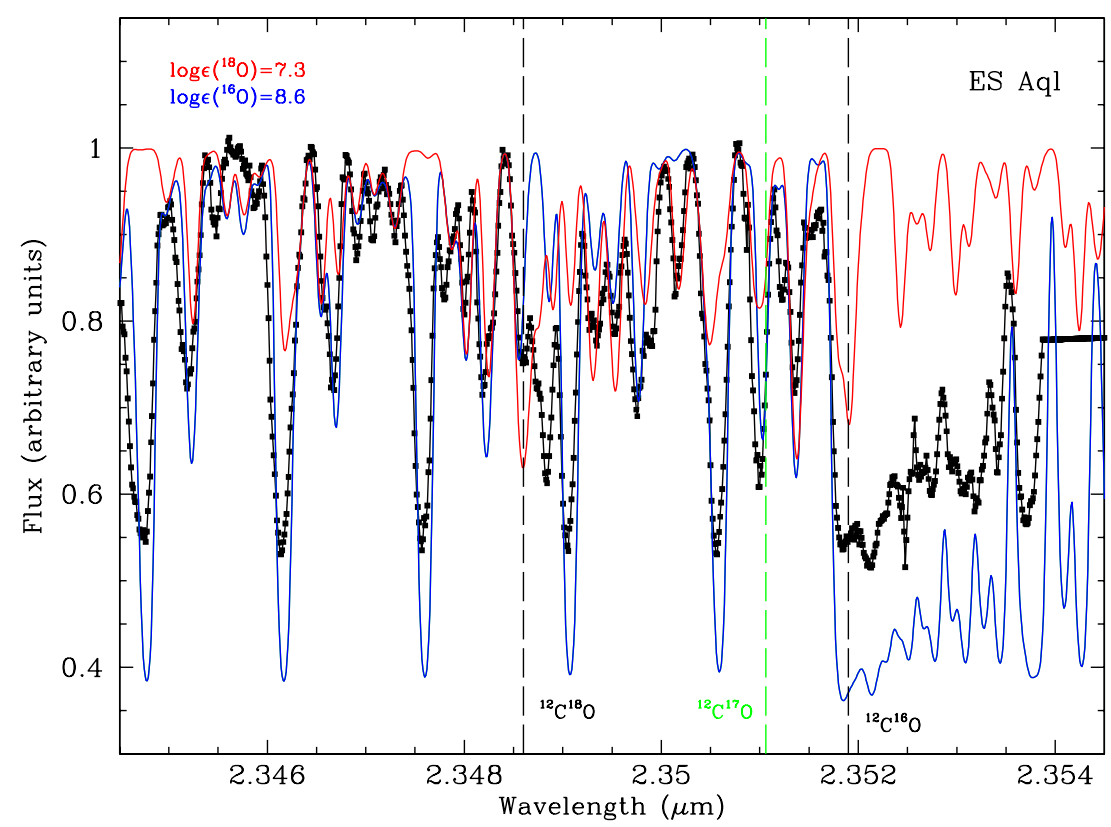

Figure 8. Synthetic spectra for the different ${ }^{12} \mathrm{C}^{16} \mathrm{O}$ and ${ }^{12} \mathrm{C}^{18} \mathrm{O}$ isomers (blue and red, respectively) and observed (black) spectrum (on 2008 April 8) in the region around the 2-0 $2.349 \mu \mathrm{m}^{12} \mathrm{C}^{18} \mathrm{O}$ band head for the RCB star ES Aql. Note that the synthetic spectra also include $\mathrm{CN}$ lines. The ${ }^{12} \mathrm{C}^{16} \mathrm{O}$ lines are saturated for this star with $T_{\text {eff }}=5000 \mathrm{~K}$. It should be noted that the synthetic blue spectrum predicts stronger ${ }^{12} \mathrm{C}^{16} \mathrm{O}$ lines than observed because the observed spectrum is diluted by circumstellar dust (see the text). The synthetic spectra are constructed by using the input $\mathrm{C}$ abundance of 9.52 and the $\mathrm{N}$ abundance of 8.6. The blue and red synthetic spectra correspond to the oxygen isotopic abundances of $\log \varepsilon\left({ }^{16} \mathrm{O}\right)=8.6$ and $\log \varepsilon\left({ }^{18} \mathrm{O}\right)=7.3$, respectively (or the isotopic ratio ${ }^{16} \mathrm{O} /{ }^{18} \mathrm{O}=20$ ).

(A color version of this figure is available in the online journal.)

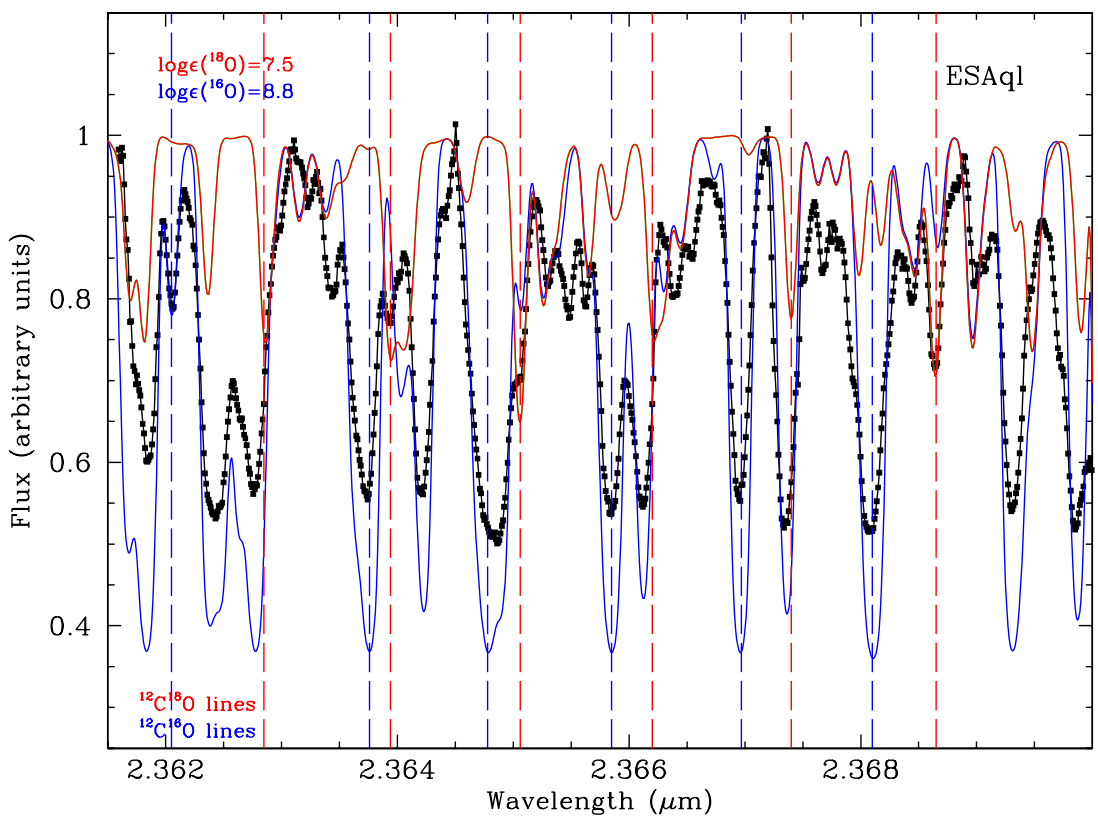

Figure 9. Synthetic spectra for the different ${ }^{12} \mathrm{C}^{16} \mathrm{O}$ and ${ }^{12} \mathrm{C}^{18} \mathrm{O}$ isomers (blue and red, respectively) and observed (black) spectrum (on $2008 \mathrm{March} 21$ ) in the wavelength region centered around $2.365 \mu \mathrm{m}$ for the RCB star ES Aql. Note that the synthetic spectra also include CN lines. As in Figure 8, the synthetic blue spectrum predicts stronger ${ }^{12} \mathrm{C}^{16} \mathrm{O}$ lines than observed because the observed spectrum is diluted by circumstellar dust (see the text). The synthetic spectra are constructed by using the input $\mathrm{C}$ abundance of 9.52 and the $\mathrm{N}$ abundance of 8.6. The blue and red synthetic spectra correspond to the oxygen isotopic abundances of $\log \varepsilon\left({ }^{16} \mathrm{O}\right)=8.8$ and $\log \varepsilon\left({ }^{18} \mathrm{O}\right)=7.5$, respectively (or the isotopic ratio ${ }^{16} \mathrm{O} /{ }^{18} \mathrm{O}=20$ ). The relative strength of the weak ${ }^{12} \mathrm{C}^{18} \mathrm{O}$ and ${ }^{12} \mathrm{C}^{16} \mathrm{O}$ lines at $\sim 2.3686 \mu \mathrm{m}$ and $\sim 2.3620 \mu \mathrm{m}$ provides an estimation of the ${ }^{16} \mathrm{O} /{ }^{18} \mathrm{O}$ in this star. The strongest ${ }^{12} \mathrm{C}^{18} \mathrm{O}$ and ${ }^{12} \mathrm{C}^{16} \mathrm{O}$ lines-most of them not useful for abundance estimations-are marked with red and blue vertical dashed lines, respectively.

(A color version of this figure is available in the online journal.)

nuclear energy release at the $\mathrm{C}-\mathrm{O}$ WD. Yet, it is very likely that a supergiant product of a merger spends most of its time as a cool star. If an $\mathrm{HdC}$ results, the evolutionary sequence will be $\mathrm{HdC} \rightarrow \mathrm{RCB} \rightarrow$ EHe. Perhaps, some mergers do not populate the coolest parts of the supergiant track, then the sequence will be $\mathrm{RCB} \rightarrow$ EHe. In other words, all $\mathrm{HdC}$ may evolve to RCB and EHe stars, but, perhaps, not all RCB and EHe stars were $\mathrm{HdC}$ stars. Here, we examine the abundances in light of the assumption that $\mathrm{HdC} \rightarrow \mathrm{RCB} \rightarrow \mathrm{EHe}$ is the common sequence. 


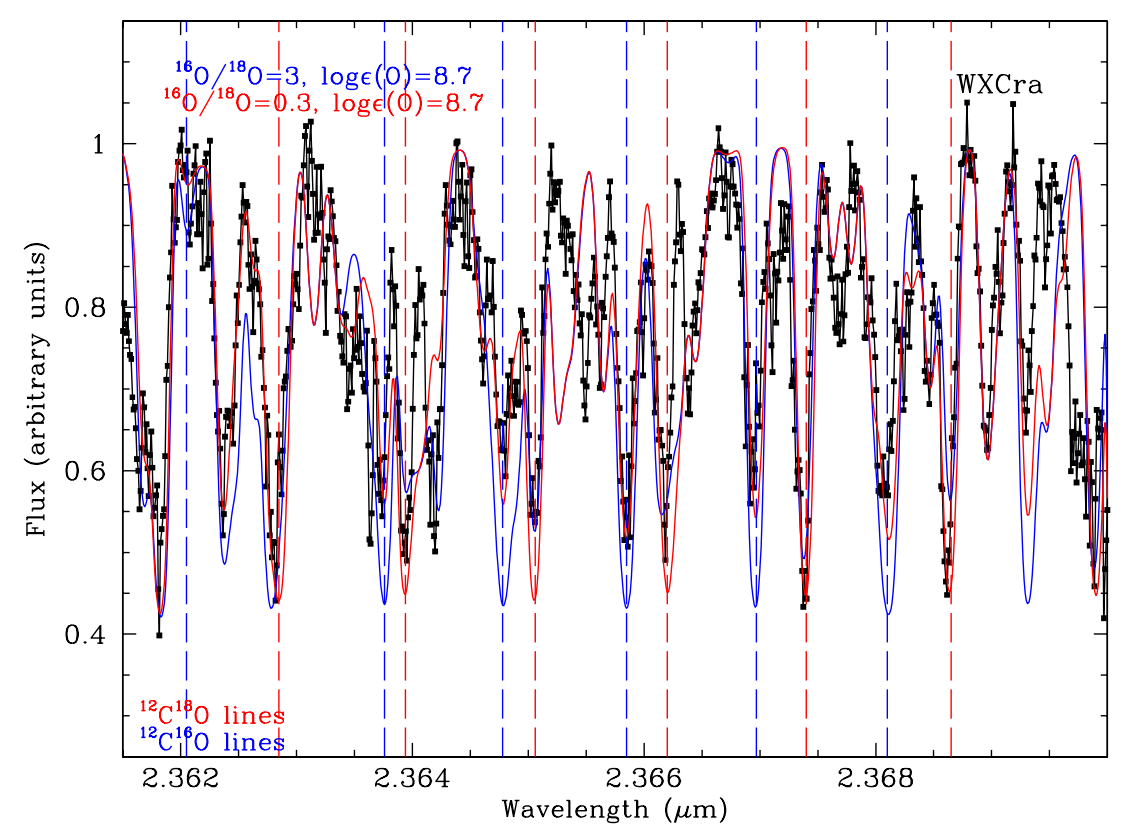

Figure 10. Best synthetic (red) and observed (black) spectra for the region centered at $2.365 \mu \mathrm{m}$ for the RCB star WX CrA. The red spectrum assumes $T_{\text {eff }}=$ $5300 \mathrm{~K}$, the input $\mathrm{C}$ abundance of 9.52 , the $\mathrm{N}$ abundance of 9.3 , a total $\mathrm{O}\left({ }^{16} \mathrm{O}+{ }^{18} \mathrm{O}\right)$ of $\log \varepsilon(\mathrm{O})=8.7$, and the isotopic ratio ${ }^{16} \mathrm{O} /{ }^{18} \mathrm{O}=0.3$. Selected ${ }^{12} \mathrm{C}^{18} \mathrm{O}$ and ${ }^{12} \mathrm{C}^{16} \mathrm{O}$ lines are marked with red and blue vertical dashed lines, respectively. A synthetic spectrum (blue) for the same total $\mathrm{O}\left({ }^{16} \mathrm{O}+{ }^{18} \mathrm{O}\right)$ of $\log \varepsilon(\mathrm{O})=8.7$ but a higher ${ }^{16} \mathrm{O} /{ }^{18} \mathrm{O}$ ratio of 3 (i.e., a lower ${ }^{18} \mathrm{O}$ abundance) is shown for comparison.

(A color version of this figure is available in the online journal.)

Table 3

Chemical Abundances in HdC and Cool RCB Stars ${ }^{\mathrm{a}}$

\begin{tabular}{lcccccc}
\hline \hline \multicolumn{1}{c}{ Star } & $\log \varepsilon(\mathrm{N})$ & $\log \varepsilon(\mathrm{O})$ & $\log \varepsilon\left({ }^{16} \mathrm{O}\right)$ & $\log \varepsilon\left({ }^{18} \mathrm{O}\right)$ & $\log \varepsilon(\mathrm{Na})$ & $\log \varepsilon(\mathrm{S})$ \\
\hline ES Aql & 8.6 & 8.6 & 8.6 & 7.3 & $\ldots$ & 6.8 \\
SV Sge & 7.7 & 8.4 & 8.4 & 7.2 & $\ldots$ & 7.6 \\
S Aps & 9.2 & 9.2 & 9.2 & 8.0 & $\ldots$ & 6.8 \\
WX CrA & 9.3 & 8.7 & 8.1 & 8.6 & 7.3 & $\ldots$ \\
U Aqr & 9.4 & 9.3 & 9.2 & 8.6 & $\leqslant 5.4$ & 6.5 \\
HD 137613 & 9.4 & 8.7 & 8.3 & 8.6 & 6.9 & 7.6 \\
HD 175893 & 9.2 & 8.7 & 8.1 & 8.6 & 6.5 & 7.6 \\
HD 182020 & 9.2 & 8.0 & 7.6 & 7.9 & 6.8 & 7.4 \\
\hline
\end{tabular}

Note. ${ }^{\text {a }}$ The first five objects are the cool RCB stars analyzed in this paper while the last three ones are the HdC stars studied by García-Hernández et al. (2009). The abundances are normalized to $\log \sum \mu_{i} \epsilon_{i}=12.15$, i.e., $\log \varepsilon(\mathrm{He})=11.52$ and $\log \varepsilon(\mathrm{C})=9.52$.

It is assumed for the purposes of this discussion that the remarkable high concentration of ${ }^{18} \mathrm{O}$ is created during or shortly after the merger. Although it is plausible that the ${ }^{18} \mathrm{O}$ is synthesized from ${ }^{14} \mathrm{~N}$ (Warner 1967), no explanation has yet been provided as to why the ${ }^{16} \mathrm{O} /{ }^{18} \mathrm{O}$ ratio takes the low values seen in the $\mathrm{HdCs}$ and why surviving ${ }^{14} \mathrm{~N}$ seems so slightly depleted. These questions were explored by Clayton et al. (2007) and García-Hernández et al. (2009). Here, we set such matters aside and discuss how the difference in ${ }^{16} \mathrm{O} /{ }^{18} \mathrm{O}$ ratios between $\mathrm{HdC}$ and $\mathrm{RCB}$ stars might be explained.

Abundances derived from the infrared spectra for the $\mathrm{HdC}$ and cool RCBs betray some intriguing differences between the two groups of $\mathrm{H}$-deficient stars. Table 3 gives the $\mathrm{N}, \mathrm{O}$ and ${ }^{16} \mathrm{O}$ and ${ }^{18} \mathrm{O}, \mathrm{Na}$, and $\mathrm{S}$ abundances for stars from this and our previous paper. The $\mathrm{C}$ abundance is assumed to be the same for all stars (see Table 2). These differences are unlikely to be attributable to systematic differences in atmospheric parameters between the $\mathrm{HdC}$ and RCB stars; the differences on average are slight except, perhaps, for the $\mathrm{C} / \mathrm{He}$ ratio which is an assumed, not a derived, parameter (see below for our conjecture about this ratio).

The most striking difference between $\mathrm{HdC}$ and RCB stars is seen among the estimates of the ${ }^{16} \mathrm{O} /{ }^{18} \mathrm{O}$ ratios. The three $\mathrm{HdCs}$ for which the ratio is measurable have an isotopic ratio near 0.5. Three of the four RCBs with a measured isotopic ratio have a ratio at least 1 order of magnitude higher than that seen in the HdCs. The exception is the RCB WX CrA which has an HdC-like ratio. It bears repeating (see Table 2) that the measured ${ }^{16} \mathrm{O} /{ }^{18} \mathrm{O}$ ratio is insensitive to the adopted model atmosphere over quite wide variations of the model atmosphere parameters. Furthermore, this HdC-RCB difference is evident from inspection of the spectra.

In order to interpret this clear difference in the ${ }^{16} \mathrm{O} /{ }^{18} \mathrm{O}$ ratio between $\mathrm{HdC}$ and $\mathrm{RCB}$ stars, it is helpful to know the contributions from an increase in the ${ }^{16} \mathrm{O}$ and a decrease in the ${ }^{18} \mathrm{O}$ abundance between the two groups of stars. The ${ }^{16} \mathrm{O}$ and ${ }^{18} \mathrm{O}$ abundances are given in Table 3 and shown in Figure 11. One interpretation of the abundances is that the difference in ${ }^{16} \mathrm{O} /{ }^{18} \mathrm{O}$ ratios may be partially attributable to decrease in ${ }^{18} \mathrm{O}$ 


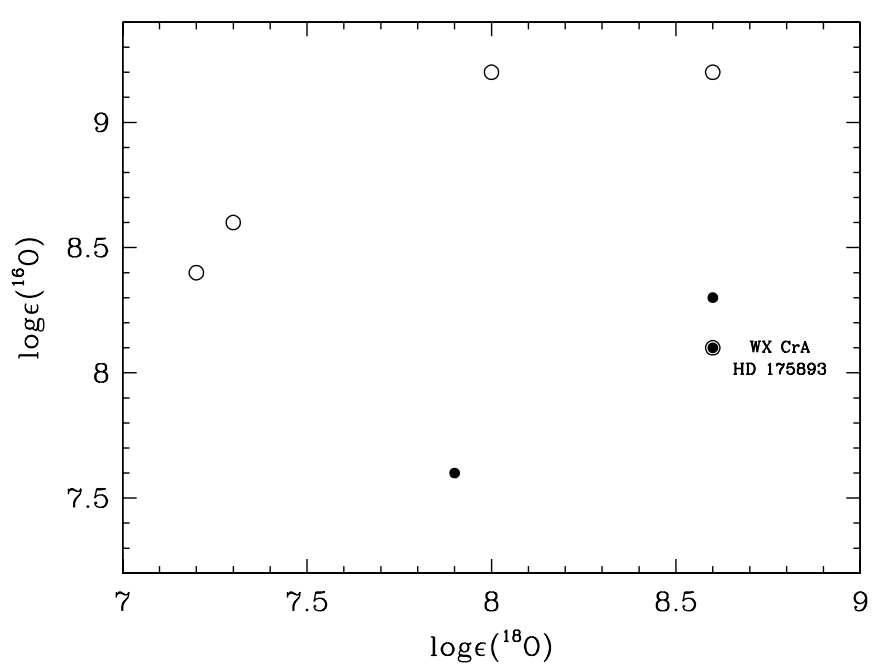

Figure 11. ${ }^{16} \mathrm{O}$ abundance vs. ${ }^{18} \mathrm{O}$ abundance for $\mathrm{HdC}$ (filled circles) and cool $\mathrm{RCB}$ (unfilled circles) stars. Note that the $\mathrm{O}$ isotopic abundances of the $\mathrm{HdC}$ star HD 175893 and the cool RCB WX CrA are identical.

abundances between the ${ }^{18} \mathrm{O}$-rich $\mathrm{HdC}$ stars (and WX CrA) but primarily arises from an increase in the ${ }^{16} \mathrm{O}$ abundance between $\mathrm{HdC}$ and RCB stars. (The lower than average ${ }^{16} \mathrm{O}$ and ${ }^{18} \mathrm{O}$ abundances for ES Aql among the RCBs may possibly be due to the uncertain and varying dilution of the spectrum by dust emission.)

Here, we do not attempt to explain the origin of the ${ }^{18} \mathrm{O}$ that is such a remarkable characteristic of the $\mathrm{HdC}-$ see Clayton et al. (2007) and García-Hernández et al. (2009) for speculations. In this section, we directly speculate how the differences in composition between $\mathrm{HdC}$ and RCB stars may be related to the dredge-up of nuclear-processed material by the supergiant as it evolves from an $\mathrm{HdC}$ to an $\mathrm{RCB}$ star. Published calculations bounding the possibilities have yet to be reported. Our speculations draw on the few models presented by Saio \& Jeffery (2002) for mergers in which mass transfer is a slow process continuing well into the supergiant phase. In these models, the convective envelope just prior to the rapid evolution back to high temperatures makes a close approach to the Heburning shell. (This close approach may not occur in the case of a merger which results in traces of hydrogen in the accreted material and then the maintenance of an H-burning shell in the supergiant.)

With a close approach to the He-burning shell, the ${ }^{16} \mathrm{O}$ in the envelope may increase. Such an increase is more certain to occur, if after the cessation of He burning, the convective envelope is able to penetrate to the He-shell/CO-core boundary. Also, the ${ }^{18} \mathrm{O}$ abundance is likely to decrease because it may have been destroyed in the deepest layers; ${ }^{18} \mathrm{O}$ is more prone to $(\alpha, \gamma)$ destruction than ${ }^{16} \mathrm{O}$ by several orders of magnitude. A few percent addition of mass from the He-exhausted layers should suffice to increase the ${ }^{16} \mathrm{O}$ abundance by an order of magnitude.

In this scenario, the ${ }^{12} \mathrm{C}$ abundance of the envelope and atmosphere increases too between $\mathrm{HdC}$ and RCB stars because the ratio of ${ }^{12} \mathrm{C} /{ }^{16} \mathrm{O}$ in the He-exhausted layers is most likely to be greater than 1 . The He abundance should not be significantly changed by the extension of the deep convective envelope across the He-shell/C-O-core boundary. Thus, the RCBs may have a higher $\mathrm{C} / \mathrm{He}$ ratio than the HdCs. This may account also for an intriguing difference in $\mathrm{Na}$ and $\mathrm{S}$ abundances between $\mathrm{HdC}$ and
RCB stars in Table 3. Both $\mathrm{S}$ and $\mathrm{Na}$ are about 0.6 dex higher in HdCs than in the RCBs.

Derived abundances are dependent on the assumed $\mathrm{C} / \mathrm{He}$ ratio. In the infrared $\mathrm{He}^{-}$free-free opacity is the leading contributor to continuous opacity with $\mathrm{C}$ as an important electron donor. In our previous paper, we reported that a change in the $\mathrm{C} / \mathrm{He}$ ratio from $1 \%$ to $10 \%$ leads to an increase of $\mathrm{Na}$ and $\mathrm{S}$ abundances by about $0.6 \mathrm{dex}$. This suggests that decreasing the $\mathrm{C} / \mathrm{He}$ ratio to about $0.1 \%$ will reduce the $\mathrm{Na}$ and $\mathrm{S}$ abundances to about their levels seen in the RCB stars. Derived abundances for $\mathrm{N}$ and $\mathrm{O}$ change little with a change in the $\mathrm{C} / \mathrm{He}$ ratio. The $\mathrm{C} / \mathrm{He}$ ratio of $1 \%$ for the $\mathrm{HdCs}$ and $\mathrm{RCBs}$ is an assumed value. The change of the $\mathrm{C} / \mathrm{He}$ ratio from $0.1 \%$ to $1 \%$ to reconcile $\mathrm{Na}$ and $\mathrm{S}$ abundances in $\mathrm{HdC}$ with values for RCBs is possibly consistent with the $\mathrm{C} / \mathrm{O}$ ratio of material dredged-up from the He-exhausted layers.

Although this scenario offers a scheme for accounting for the lowering of the ${ }^{16} \mathrm{O} /{ }^{18} \mathrm{O}$ ratio between $\mathrm{HdC}$ and RCB stars and also for the difference in their abundances of $\mathrm{Na}$ and $\mathrm{S}$, it augments the challenge in accounting for the compositions of the warm RCBs (and EHes) for which detailed results are available (Asplund et al. 2000; Pandey et al. 2006). If the RCBs are affected by a late dredge-up, one would suppose that the final $\mathrm{C} / \mathrm{He}$ must vary from star to star on account of the different amounts of mass dredged up and the different $\mathrm{C} / \mathrm{O}$ ratios. Such a variation would be reflected in star-to-star variations in abundances when all stars are analyzed assuming the same $\mathrm{C} / \mathrm{He}$ ratio. Yet, the abundances of elements heavier than $\mathrm{O}$ are quite similar star-to-star across the sample of majority RCBs. The dispersion including the measurement uncertainties is only about 0.2 dex for well-observed elements over the sample of 14 warm RCBs.

The challenge in accounting for the uniform compositions was not created by incorporating a late dredge-up into the evolution of these $\mathrm{H}$-deficient supergiants. Its origin surely lies in the DD scenario itself. Mergers of He WDs with $\mathrm{C}-\mathrm{O}$ WDs spanning a range in masses and previous histories of the WDs might be expected to result in $\mathrm{H}$-deficient stars with quite different $\mathrm{C} / \mathrm{He}$ ratios and metallicities.

One looks forward to detailed modeling of $\mathrm{H}$-deficient supergiants that will test our conjecture about a late dredge-up of material from $\mathrm{He}$-exhausted layers.

\section{CONCLUDING REMARKS}

Challenges are not a new phenomenon to students of the RCB stars. High-resolution infrared spectroscopy promises to provide responses to some of the key challenges regarding the $\mathrm{RCBs}$ and their putative relatives the $\mathrm{HdC}$ and EHe stars. Here, we suggested an evolutionary link between the $\mathrm{HdCs}$ and the RCBs involving the dredge-up of material to the surface. Further exploration of the link is possible. Unfortunately, the list of known HdCs has been exhausted. A few cool RCBs remain to be observed in the $K$ band at high resolution. Of these, the most critical is V CrA. Unfortunately, our observations were obtained when the star was at minimum and infrared emission came from the dust shell and not the stellar photosphere. V CrA is the special target because it is one of four known minority RCB stars (Rao \& Lambert 1994) and the only one likely to show CO lines. Minority RCBs are distinguished by their high $\mathrm{S} / \mathrm{Fe}$ and $\mathrm{Si} / \mathrm{Fe}$ ratios (among other abundance anomalies): $\mathrm{V} \mathrm{CrA}$ has $[\mathrm{Si} / \mathrm{Fe}] \simeq[\mathrm{S} / \mathrm{Fe}] \simeq 2$ (Asplund et al. 2000; Rao \& Lambert 2008). Additionally, V CrA is rich in ${ }^{13} \mathrm{C}$ with ${ }^{12} \mathrm{C} /{ }^{13} \mathrm{C} \simeq 3$ (Rao \& Lambert 2008). Among those $\mathrm{HdC}$ and 
RCBs for which the carbon isotopic ratio may be estimated, $\mathrm{V} \mathrm{CrA}$ is the only known example with a high ${ }^{13} \mathrm{C}$ abundance. What does the combination of anomalous abundance ratios and high ${ }^{13} \mathrm{C}$ abundance imply about the origin of V CrA and minority RCBs? Is their origin a variant of the DD scenario or must another (i.e., the FF) scenario be invoked? Measurement of the oxygen isotopic ratios is potentially a valuable clue to the answers to these questions.

Infrared spectroscopy offers the possibility of probing the carbon problems posed by the RCBs and $\mathrm{HdCs}$ - see Asplund et al. (2000) and García-Hernández et al. (2009)—in the failure to account for the strengths of the atomic and molecular carbon lines. From the optical to the infrared, the leading contributor to the continuous opacity depending on the star's effective temperature may change from the photoionization of neutral carbon to free-free absorption from neutral helium. Full spectral coverage at high spectral resolution from the optical through at least the $K$ band offers a novel opportunity to probe and hopefully to resolve the carbon problems. With their resolution, the chemical compositions of these fascinating H-deficient stars should be on a firmer footing and insights should be gained into their origins.

D.A.G.H. acknowledges support for this work provided by the Spanish Ministry of Science and Innovation (MICINN) under the 2008 Juan de La Cierva Program and under grant AYA-2007-64748. D.A.G.H. also acknowledges the great hospitality of Dr. N. Kameswara Rao during his stay at the Indian Institute of Astrophysics (Bangalore, India). This paper is based on observations obtained at the Gemini Observatory, which is operated by the Association of Universities for Research in Astronomy, Inc., under a cooperative agreement with the NSF on behalf of the Gemini partnership: the National Science Foundation (United States), the Science and Technology Facilities Council (United Kingdom), the National Research Council (Canada), CONICYT (Chile), the Australian Research
Council (Australia), Ministério da Ciência e Tecnologia (Brazil), and Ministerio de Ciencia, Tecnología e Innovación Productiva (Argentina). The observations were obtained with the Phoenix infrared spectrograph developed by the National Optical Astronomy Observatory. The spectra were obtained as part of program GS-2008A-Q-11. This research has been supported in part by grant F-634 to D.L.L. from the Robert A. Welch Foundation of Houston, Texas. K.E. gratefully acknowledges support from the Swedish Research Council.

\section{REFERENCES}

Alvarez, R., \& Plez, B. 1998, A\&A, 330, 1109

Asplund, M., Gustafsson, B., Kiselman, D., \& Eriksson, K. 1997, A\&A, 318, 251

Asplund, M., Gustafsson, B., Lambert, D. L., \& Rao, N. K. 2000, A\&A, 353, 287

Asplund, M., Gustafsson, B., Rao, N. K., \& Lambert, D. L. 1998, A\&A, 332, 651

Blöcker, T. 2001, Ap\&SS, 275, 1

Bond, H. E., Luck, R. E., \& Newman, M. J. 1979, ApJ, 233, 205

Clayton, G. C., Geballe, T. R., Herwig, F., Fryer, C., \& Asplund, M. 2007, ApJ, 662,1220

Clayton, G. C., Herwig, F., Geballe, T. R., Asplund, M., Tenenbaum, E. D., Engelbracht, C. W., \& Gordon, K. D. 2005, ApJ, 623, L141

Cottrell, P. L., \& Lawson, W. A. 1998, PASA, 15, 179

García-Hernández, D. A., Hinkle, K. H., Lambert, D. L., \& Eriksson, K. 2009, ApJ, 696, 1733

Herwig, F. 2001, Ap\&SS, 275, 15

Hinkle, K. H., et al. 2003, Proc. SPIE, 4834, 353

Iben, I. J., \& Tutukov, A. V. 1984, ApJS, 54, 335

Lawson, W. A., Cottrell, P. L., Kilmartin, P. M., \& Gilmore, A. C. 1990, MNRAS, 247,91

Pandey, G., Lambert, D. L., Jeffery, C. S., \& Rao, N. K. 2006, ApJ, 638, 454

Rao, N. K. 2008, in ASP Conf. Ser. 391, Hydrogen Deficient Stars, ed. K. Werner \& T. Rauch (San Francisco, CA: ASP), 25

Rao, N. K., \& Lambert, D. L. 1994, JA\&A, 15, 47

Rao, N. K., \& Lambert, D. L. 2008, MNRAS, 384, 477

Saio, H., \& Jeffery, C. S. 2002, MNRAS, 333, 121

Warner, B. 1967, MNRAS, 137, 119

Webbink, R. F. 1984, ApJ, 277, 355 